\title{
Contribuições Teóricas e Metodológicas da Abordagem Multinível para o Estudo da Aprendizagem e sua Transferência nas Organizações
}

\author{
Maria Júlia Pantoja \\ Jairo Eduardo Borges-Andrade
}

\begin{abstract}
Resumo
Este ensaio inicialmente discute os conceitos de aprendizagem e de transferência de aprendizagem nas organizações, partindo das controvérsias teóricas existentes na psicologia e dos usos desses termos na linguagem cotidiana. Depois, sintetiza os princípios da abordagem multinível para a construção de teoria e a realização de pesquisa nas organizações e demonstra como eles podem ser aplicados para a compreensão da transferência de aprendizagem decorrente dos processos de treinamento e desenvolvimento. Faz então um levantamento da produção nacional de pesquisas sobre transferência de aprendizagem nas organizações, mostrando como têm sido realizadas as medidas de transferência e identificados os seus preditores. Em seguida, destaca alguns aspectos metodológicos de algumas delas, escolhidas com base no critério da diversidade das medidas e dos preditores investigados. Finalmente, sugere como estudos futuros poderiam levar em conta a abordagem multinível.
\end{abstract}

Palavras-chave: aprendizagem; transferência de aprendizagem; treinamento e desenvolvimento; abordagem multinível.

\section{Abstract}

This analysis starts discussing the concepts of learning and transfer of learning into organizations, according to the theoretical controversies which are inserted in psychology and the use of these terms in the daily language. Afterwards, it synthesizes the principles of the multilevel approach to build up a theory and to accomplish research into organizations and shows how they can be used for understanding the transfer of learning that results from training and development processes. Then, it makes a survey of the national research production on learning transfer into organizations, showing how the measures of learning transfer into organizations have been done and how their predictors have been identified. Following, some methodological issues of part of this research is highlighted, based on two criteria: diversity of measurement and of studied predictors. Finally it suggests how future studies could take into account the multilevel approach.

Key words: learning; learning transfer; training and development; multilevel approach. 


\section{Aprendizagem e Transferência nas Organizações}

O presente texto tem como objetivo sugerir o uso da abordagem multinível, proposta no livro de Klein e Kozlowski (2000), para o estudo da transferência de aprendizagem nas organizações. Neste livro (páginas 3 e 163), são apresentados dois axiomas que precisam ser aqui lembrados, pois orientarão o restante deste ensaio. O primeiro deles é que as organizações são sistemas multiníveis, que integram processos que podem ocorrer nos níveis individual, grupal e organizacional. O segundo é que a aprendizagem é processo psicológico que ocorre no primeiro desses níveis; portanto, no sentido preciso e primordial desse processo e contrariando certa literatura contemporânea em administração, equipes e organizações não aprendem.

Para compreender como o aprendido no primeiro nível pode produzir seus efeitos neste nível, será preciso lançar mão do conceito de transferência e do que foi sobre ele estudado pela psicologia da aprendizagem, desde o início do século XX. Para melhor compreender como podem ocorrer os efeitos nos outros dois níveis, serão também necessários os conceitos e as hipóteses elaboradas no âmbito da teoria multinível desenvolvida pela psicologia organizacional no final do referido século. Nos próximos parágrafos e na seção seguinte serão extraídas as informações essenciais que servirão de base para o conjunto de argumentos e sugestões que serão apresentados no restante do ensaio. Essa extração foi feita do livro acima mencionado e do texto de autoria de Abbad e Borges-Andrade (2004), que detalham o posicionamento teórico, sobre a aprendizagem e sua transferência, do grupo de pesquisa ao qual pertencem os autores do presente ensaio.

O termo aprendizagem possui ampla variedade de definições em psicologia, dependendo da teoria que o autor abraça ${ }^{(1)}$. De forma geral, o referido termo faz referência a um processo de mudanças que ocorre no indivíduo e que não é resultante de maturação, uma expressão verbal concernente a outro conceito que está associado à idade ou fases da vida. Essas mudanças geralmente perduram ao longo do tempo. Para diferenciar a aprendizagem de outros processos, as definições geralmente qualificam essas mudanças como sendo resultado da experiência do indivíduo. Na tradição behaviorista (teorias S-R), a ênfase é colocada na mudança de comportamento (R), que se estabelece de forma relativamente duradoura, como resultado da interação do indivíduo com seu ambiente (S). Na tradição cognitivista (teorias S-O-R), aquela mudança duradoura de comportamento $(\mathrm{R})$ também ocorreria como resultado desta 
interação com o ambiente (S); mas é postulado que a interação antes resultaria em processos mentais ou na aquisição de conhecimentos, habilidades e atitudes CHAs $(\mathrm{O})$ que poderiam ser inferidos a partir daquelas mudanças.

Na linguagem comum (ver, por exemplo, os dicionários Aurélio, Caldas Aulete e Koogan/Houaiss), a aprendizagem ou o ato de aprender está geralmente associado às noções de adquirir, tomar, reter, segurar, pegar, agarrar, prender e assimilar. Isto é, nele está quase sempre embutido o sentido figurado da apropriação ou da apreensão. Tal como na abordagem cognitivista de aprendizagem, a experiência de interação do indivíduo com seu ambiente (S) lhe possibilitaria apreender algo (O) - uma capacidade (por exemplo, um conceito ou uma forma de resolver um problema) ou uma disposição (por exemplo, um interesse ou um valor), que seria futuramente mostrado, manifestado, evidenciado ou revelado através de alguma mudança em seu comportamento $(\mathrm{R})$. O ensaio a ser aqui apresentado tem por base as teorias S-O-R de aprendizagem.

Se a mudança no indivíduo ocorre em atividades não equivalentes às anteriores ou é verificada em situações distintas daquelas em que ocorreu a aquisição, pode ser dito que ocorreu uma transferência de aprendizagem. Outra consulta aos mencionados dicionários irá sugerir que o termo transferir freqüentemente corresponde às noções de passar, trocar, substituir, transmitir, transpor, deslocar, atravessar e transportar, isto é, aquilo que foi antes adquirido pode ser depois cedido. O sentido é metafórico, como bem lembrou Abbad-OC (1999), ao alertar para o fato de que na realidade a transferência não é coisa, mas conceito em psicologia.

Quando a transferência de aprendizagem é estudada nas organizações, as medidas realizadas geralmente fazem referência ao uso ou aplicação, no trabalho, do que foi anteriormente aprendido. Este uso ou aplicação pode ser considerado uma instância de comportamento organizacional, tal como todas as outras: por exemplo, as relativas aos constructos motivação, satisfação ou comprometimento no trabalho ou liderança na organização, que são usadas nesta subárea de conhecimento da administração ou da psicologia. Será por meio dessas medidas que o estudioso poderá fazer inferências sobre o desenvolvimento de processos mentais ou sobre CHAs que teriam sido aprendidas e transferidas em contextos organizacionais.

As primeiras teorias de transferência de aprendizagem fizeram convergir seu interesse para o número de elementos idênticos nas situações e nos comportamentos, antes e depois da aprendizagem, para explicar sua transferência. Isso pode ser constatado em livros textos que descrevem teorias S-R e que fazem referências a mudanças de comportamento e às generalizações de estímulos e 
de respostas ou ao controle de estímulos. Nas teorias S-O-R, a organização do conhecimento que o aprendiz traz consigo passou a ter importância equivalente ou superior àquela dada para as possíveis similaridades ou diferenças existentes entre situações ou entre comportamentos, para explicar os processos de transferência.

Para aumentar a probabilidade de ocorrer transferência de aprendizagem, o trabalhador deve ser exposto a uma variedade de contextos e de atividades; e as informações a serem por ele armazenadas devem ser organizadas de forma que possam ser facilmente recuperadas e futuramente utilizadas em novas tarefas ou situações organizacionais. Gage e Berliner (1984) argumentam que metacognições (habilidades de automonitoramento, auto-regulação e auto-gestão), relacionadas à supervisão que o próprio aprendiz faz sobre sua aquisição de CHAs e a aplicação em outros contextos promovem essa transferência. Além disso, Gagné e Driscoll (1988) sugerem que o desenvolvimento pleno de competências dos níveis inferiores da hierarquia de aprendizagem facilitaria a aprendizagem de competências dos níveis superiores desta mesma hierarquia: por exemplo, o domínio de conceitos seria transferido para o da aplicação de regras; o domínio da aplicação de regras facilitaria o de solução de problemas.

Do mesmo modo que em qualquer outra esfera da vida, os indivíduos em seu trabalho podem aprender: por exemplo, por meio das conseqüências organizacionais resultantes de seus comportamentos; observando as conseqüências dos comportamentos dos demais membros de sua equipe; ouvindo histórias de seus companheiros mais antigos; ou recebendo instruções de seus supervisores ou de outras pessoas designadas pela organização. Essa aquisição pode ou não levar a desempenhos melhores que os anteriores; pode ou não ser consciente ou deliberada; e pode envolver atos explícitos ou implícitos. O aprendido durante a realização de uma tarefa de trabalho, ou em contato com outras pessoas, ou em interação com uma máquina pode ou não ser transferido para outros contextos ocupacionais ou organizacionais. Em sua proposta, Howard (1999) define transferência de aprendizagem como sendo o uso de representações mentais declarativas (exemplos: informações, crenças, valores) e procedimentais (exemplos: categorizações, regras, soluções de problemas) formadas num ambiente, em outro ambiente a este relacionado, ou como sendo alterações de representações mentais para se ajustarem a situações relacionadas.

As pessoas podem estar o tempo todo aprendendo. Para possibilitar o estudo do fenômeno, os pesquisadores procuram assim produzir ou encontrar situações onde é possível identificar os comportamentos anteriores e posteriores à aquisição de CHAs. Nos laboratórios, isto é geralmente feito por meio do estabelecimento 
de respostas arbitrárias. Nas escolas, procura-se investigar os comportamentos dos alunos em situações de ensino. Nas organizações, os processos de treinamento e desenvolvimento (T\&D) são o foco mais usual das pesquisas sobre aprendizagem. Para posteriormente estudar nos laboratórios a transferência do aprendido, será então necessário utilizar situações ou respostas distintas das anteriormente experimentadas. Nas escolas, será preciso investigar o uso do aprendido em outras situações de ensino ou na vida do aluno. Nas organizações, será preciso buscar esse uso fora dos processos de T\&D, costumeiramente no desempenho em contextos diversificados de trabalho.

Processos de $T \& D$ podem ser definidos como ações organizacionais que utilizam uma tecnologia instrucional ou são deliberadamente arranjadas, visando à aquisição de CHAs para superar deficiências de desempenho no trabalho, ou preparar empregados para novas funções, ou adaptar mão de obra para a introdução de novas tecnologias, ou promover o livre crescimento dos membros de uma organização (Borges-Andrade, 2002). Essa é uma definição S-O-R, pois sugere que ações organizacionais (S) promovem a aquisição de CHAs (O) que se traduziriam em mudanças de comportamento (R) durante T\&D (aprendizagem) ou no trabalho (transferência de aprendizagem). As organizações investem em processos de T\&D e podem esperar várias conseqüências: (1) que as pessoas fiquem satisfeitas com esses processos; (2) que aprendam; e (3) que ocorram transferências de aprendizagem para o trabalho e transferências para o desempenho das equipes e das organizações.

O foco do presente ensaio é neste terceiro tipo de conseqüência, que ocorre em médio ou longo prazo. Quando ele diz respeito à aprendizagem, é inerente ao nível do indivíduo, mas poderia promover mudanças nos níveis de equipe e da organização. Baldwin e Ford (1988) definem transferência de treinamento como a aplicação, generalização e manutenção, no ambiente de trabalho, de CHAs adquiridos em T\&D. Contudo, no presente ensaio, este conceito continuará sendo denominado transferência de aprendizagem, já que a coisa que é transferida seriam os CHAs adquiridos e não tudo o que está incorporado no conceito de T\&D anteriormente apresentado: ações organizacionais + aquisição de CHAs + mudança de comportamento.

Birdi (2000) propõe uma taxonomia multinível para classificar os achados das pesquisas realizadas a respeito daquele terceiro tipo de conseqüência, embora seu sistema de classificação vá além do que poderia ser considerado como transferência de aprendizagem. Segundo este autor, T\&D podem ter efeitos nos níveis do indivíduo, da equipe e da organização. Ao utilizar os três mencionados níveis, a taxonomia passa a ser um complemento oportuno para a abordagem multinível, proposta no livro de Klein e Kozlowski (2000), mesmo não tendo ocorrido 
qualquer contato entre os autores mencionados e ainda que tudo indique que um desconhecesse o que os outros faziam ${ }^{(2)}$.

A seguir, são descritos alguns exemplos do que foi categorizado por Birdi (2000) e que pode ser aqui arrolado como possivelmente pertencente àquele terceiro tipo de conseqüência.

\section{. Efeitos no nível do indivíduo}

Afetivos: atitudes frente a pessoas, objetos e aspectos do trabalho, atitudes sobre o não trabalho, sobre si próprio e sobre a aprendizagem; motivação no trabalho; sentimento de auto-eficácia; percepção de bem-estar mental; trabalho em rede e em colaboração com outras pessoas, capacitação de outras pessoas.

Cognitivos: uso de informações verbais, conceitos e regras no trabalho, solução de problemas ou criatividade na realização de tarefas.

Psicomotores: velocidade e fluidez no desempenho do trabalho.

\section{. Efeitos no nível da equipe}

Afetivos: coesão, satisfação e auto-eficácia da equipe e sua orientação para a tarefa, ambigüidade de papéis na equipe.

Cognitivos: conhecimento possuído pelo conjunto de membros da equipe e representado mentalmente pelo conjunto desses membros.

De desempenho: comunicação interna, divisão de responsabilidades, solução de problemas e tomada de decisão na equipe e níveis de participação dos membros da equipe.

De qualificação: certificações formais recebidas pela equipe, tais como as de qualidade.

\section{. Efeitos no nível da organização}

Relativos à aquisição de recursos: novos clientes, compra de outras organizações.

Relativos a processos internos: novas tecnologias de trabalho, clima organizacional, absenteísmo e rotatividade do conjunto de trabalhadores, taxas de acidentes de trabalho.

Relativos a metas de produtos: quantidade, qualidade e variedade do que foi produzido pela organização ou suas unidades. 
Relativos a metas do sistema: crescimento e lucro da organização, retorno de investimentos em T\&D.

Efeitos constituintes: satisfação de consumidores e acionistas, imagem organizacional.

Uma análise dos efeitos anteriormente listados, em face do que foi até aqui discutido a respeito de transferência, leva imediatamente a dois questionamentos importantes. O primeiro deles é metodológico, concernente às dificuldades que existem para estabelecer relações de determinação entre esses efeitos, especialmente os dos últimos dois níveis, e os processos de T\&D. Esta questão já foi objeto de alguma preocupação, mesmo em publicações nacionais (ver BorgesAndrade, 2002). O segundo é teórico, pois toda a literatura clássica, advinda da psicologia da aprendizagem, só pode ser aplicada no primeiro nível, onde a transferência é horizontal, conforme apontam Koslowski, Brown, Weissbein, Cannon-Bowers e Salas (2000).

Salvo raras exceções, o que é sabido (ver, por exemplo, Salas e Cannon-Bowers, 2001 e Abbad, Pantoja e Pilati, 2001) a respeito de como o indivíduo adquire CHAs e depois as transfere, pode somente ser usado para compreender as transformações que acontecem em seu desempenho no trabalho, mas não o que ocorre nos níveis de equipe e de organização. Como afirmam Salas e CannonBowers (2001, p. 489), “... precisamos assumir que efeitos de aprendizagem no nível individual emergirão, para influenciar efeitos de nível mais elevado. Transferência vertical de treinamento é a próxima fronteira. Transferência vertical pode ser um ponto de alavancagem para o fortalecimento dos vínculos entre os efeitos de aprendizagem e a efetividade organizacional”. Uma teoria multinível, que pudesse simultaneamente abordar as questões de transferência horizontal, que é por natureza de aprendizagem, e vertical, cuja natureza é organizacional, seria muito útil. Por isto, ela vem resumida a seguir.

\section{A Abordagem Multinível nas Organizações}

Koslowski e Klein (2000) sintetizaram as bases teóricas, há muito conhecidas, da teoria geral de sistemas, que são essenciais para a compreensão da teoria multinível em organizações. A noção de isomorfismo, pela qual é proposto que conceitos idênticos podem ser generalizados para fenômenos de sistemas diferentes, permite imaginar que alguns fenômenos como aquisição e transferência podem ocorrer nos níveis dos indivíduos, das equipes e da organização. A de homologia afirma que processos paralelos, vinculando conceitos 
diferentes, podem ser generalizados para fenômenos de sistemas diferentes, o que permite, por exemplo, afirmar que os processos no nível individual, pelos quais as conseqüências afetam o desempenho ou pelos quais a observação da ocorrência de resultados contingentes ao desempenho de pares afeta o desempenho do observador, podem igualmente ocorrer nos níveis da equipe e da organização como um todo.

Sistemas organizacionais teriam estruturas e processos análogos; contudo a teoria geral de sistemas só teria influência metafórica, pois organizações são sistemas sociais qualitativamente distintos dos indivíduos, razão pela qual, por exemplo, talvez fosse temerário ir tão longe, a ponto de supor que a aprendizagem, tal como ela é concebida pela teoria S-O-R, pudesse ocorrer fora das pessoas. Se ela for simplesmente concebida sob o enfoque behaviorista, como mudança duradoura de comportamento resultante da interação com o ambiente (teoria SR), excluídas as inferências sobre processos mentais controlados pelo próprio indivíduo (O), talvez fosse possível usar o termo aprendizagem para designar o que ocorre com as equipes e com as organizações. A teoria geral de sistemas teria somente um valor heurístico, contribuindo em muito menor grau para o desenvolvimento de princípios testáveis no âmbito das ciências psicológica e sociais que se dedicam a estudar respectivamente os fenômenos micro e macro organizacionais.

A perspectiva micro tem seu foco nas variações entre características pessoais que afetam comportamentos do indivíduo e corre sempre o risco de negligenciar os contextos, especialmente se estes vão além daqueles que são definidos e mensurados no nível do indivíduo. Por exemplo, Rodrigues (2000) demonstrou que ex-treinandos com elevados níveis de comprometimento organizacional (característica pessoal) relatam mais intensamente os impactos de treinamentos em seu desempenho individual (comportamento organizacional micro); e que estes relatos estão fortemente associados a percepções de suporte organizacional para a transferência do aprendido para o trabalho (contexto definido e medido no nível individual). A perspectiva macro tem seu foco nos comportamentos agregados ou coletivos, ignorando a variação individual. Mas corre o risco do antropomorfismo, quando, por exemplo, importa a noção cognitivista de aprendizagem, atribuindo a níveis de natureza social uma linguagem e alguns conceitos que parecem próprios do ser humano individual.

A teoria geral de sistemas permite supor que os fenômenos micro estão embutidos em contextos macro e que estes freqüentemente emergem da interação e dinâmicas de elementos de nível inferior. Deste modo, uma abordagem multinível combinaria ambas as perspectivas, incluindo respectivamente os fenômenos topodescida (denominados top-down em inglês) e os de fundo-subida (ou bottom- 
up). Sua aplicação nas organizações seria baseada na noção interacionista de que o comportamento das pessoas no trabalho é o resultado combinado de efeitos de características pessoais (por exemplo, crenças e valores sobre o trabalho) e de contexto, incluindo neste último as suas características objetivas (por exemplo, tamanho da equipe e da organização) e interpretativas (por exemplo, natureza da liderança exercida na equipe e cultura organizacional).

Klein e Koslowski (2000) também se inspiraram em princípios, dispersos na literatura, para construir uma teoria e realizar pesquisas multiníveis em organizações. O desafio, segundo eles, é não pensar micro nem macro, mas pensar micro e macro. Seu objetivo é o de propor princípios que possibilitem uma compreensão mais integrada dos fenômenos revelados entre níveis nas organizações. O primeiro conjunto de princípios, para construir a teoria, foi resumido em termos de cinco questões centrais: Que? Como? Onde? Quando? Por que? Por que não? O segundo, para realizar pesquisa, foi descrito para sugerir a operacionalização deste elenco: constructos, níveis de medidas, tipos de modelos de investigação, amostragem e estratégias de análise de dados. A seguir, ambos os conjuntos serão apresentados e algumas considerações serão feitas no concernente à sua aplicação, para compreender a aprendizagem e sua transferência nas organizações.

Na construção de uma teoria multinível, o fenômeno e o constructo de interesse devem ser designados e definidos e serem relevantes. No presente texto isto foi realizado para os fenômenos de aprendizagem e sua transferência. Eles deveriam ser explicados por processos topo-descida ou concernentes a influências do contexto, como no estilo de liderança, exercido pelos membros da equipe, que afeta a transferência da aprendizagem em treinamentos gerenciais; ou processos fundo-subida ou de emersão. Estes processos poderiam ser de dois tipos:

· por composição, quando o fenômeno é o mesmo em todos os níveis; por exemplo, quando o número sucessivo de pessoas treinadas para atender clientes com rapidez aumenta sucessivamente os bônus de venda da equipe e, no nível seguinte, aumenta sucessivamente os lucros da empresa;

- por compilação, quando o fenômeno, no nível mais alto, é uma combinação complexa de contribuições diversas nos níveis inferiores; por exemplo, quando aumentos sucessivos no número de assistentes de instrumentação, treinados, não elevarão sucessivamente a qualidade das cirurgias realizadas pelas equipes de um hospital, pois o desempenho dessas equipes depende do treino de todos para trabalharem em conjunto e do treino de cada um em sua especialidade.

Os níveis de indivíduo, equipe e organização em que esses processos ocorrem devem ser especificados. Os vínculos entre processos serão mais fortes, se os 
níveis em que estiverem forem próximos ou interligados. Assim, a percepção de suporte psicossocial à transferência seria um preditor mais poderoso de transferência de aprendizagem, quando comparada com preditores como orientação da equipe para a tarefa ou cultura organizacional de conservação, sendo que esta última teria os menores valores de predição dessa transferência. Os processos topo-descida têm escalas de tempo menores que os processos fundo-subida. Deste modo, seriam mais rapidamente detectados os efeitos da orientação da equipe para a tarefa na aprendizagem do indivíduo em treinamento do que os efeitos desta aprendizagem no desempenho da equipe.

Os processos topo-descida e fundo-subida poderão exercer seu predomínio em diferentes períodos do ciclo de vida de fenômenos como a aprendizagem e sua transferência. Certos vínculos entre processos podem ser mais fortes em algumas épocas que em outras. Por exemplo, durante a realização de um treinamento predominarão as influências de variáveis dos níveis de equipe e da organização sobre a aprendizagem. Depois de encerrado o treinamento, esses processos poderão ser parcialmente contrabalançados por processos de emersão, sejam eles por composição ou por compilação, nos quais os CHAs adquiridos e transferidos para o desempenho do indivíduo seriam depois incorporados nos níveis de equipe e da organização.

Para a operacionalização da pesquisa multinível, o nível de cada constructo deve ser especificado. Assim, transferência de aprendizagem só ocorreria no nível do indivíduo, mas podem ocorrer transferências verticais entre este e os demais. Se os constructos dos níveis superiores forem baseados em processos de emersão, o nível de origem do constructo emergente deve ser explicitado, bem como a natureza desses processos. Se o nível do constructo for o de unidade organizacional, é preciso explicitar se suas propriedades são:

- globais, originadas neste nível, não existindo possibilidade de variarem dentro da unidade; por exemplo, o tamanho da equipe é uma propriedade global desta unidade;

- compartilhadas, emergem, por isomorfismo ou composição, de comportamentos, cognições de membros ou da interação entre eles; por exemplo, clima organizacional ou normas grupais;

- configurais, emergem como as anteriores, mas de forma descontínua ou por compilação; por exemplo, diversidade racial na organização, combinação de CHAs num treinamento.

Não existem medidas únicas ou melhores para constructos no nível de unidade: o tipo de constructo e o modelo teórico subjacente as determinam. Mas, de forma geral, pode ser dito que as propriedades: 
- globais são medidas no nível de unidade, geralmente fornecidas por informantes peritos; por exemplo, gerentes ou técnicos com acesso a indicadores relativos a essa unidade;

- compartilhadas nunca são fornecidas por tais peritos, mas sim por meio de cálculos, como o da média de respostas de vários membros da unidade, desde que o consenso, a similaridade ou a concordância tenham sido verificados;

. configurais podem ser fornecidas por peritos, desde que sejam observáveis; por exemplo, mínimo-máximo, variância, similaridade de perfil, redes e outros, mas nunca calculadas através da média.

Nos dois últimos casos, elas deveriam ser medidas no nível de origem do constructo e os dados deveriam ser tratados para refletirem a forma da emersão.

Há grande variedade de modelos teóricos testáveis na pesquisa organizacional. Eles poderiam ser sintetizados e incluídos em três categorias ${ }^{(3)}$, ilustradas a seguir:

- Modelos de um único nível. Um caso de complexidade mediana poderia ser o de uma pesquisa em que a criatividade das equipes seria predita pela diversidade de competências entre seus membros e pela quantidade de aprendizagem de CHAs ocorrida durante treinamentos de criatividade de seus gerentes.

- Modelos cruzados com efeitos diretos. Um caso complexo de modelo seria o da transferência de aprendizagem para o trabalho dos indivíduos, sendo predita pelo seu tempo de serviço, pela diversidade de tempos de serviço nas equipes e pelas políticas de retenção de pessoal das organizações participantes do estudo.

- Modelos cruzados com outros efeitos. Um caso simples poderia ser o teste da hipótese de que a existência de elevados níveis de suporte à transferência de aprendizagem nas equipes reduziria a correlação positiva entre o uso de exercícios práticos em treinamentos e a transferência da aprendizagem resultante para o desempenho dos indivíduos no trabalho.

A amostragem de dados na pesquisa multinível deve permitir variabilidade entre unidades em todos os níveis relevantes do modelo de pesquisa a ser testado. De acordo com Snijders e Bosker (1999), o tamanho da amostra é um aspecto que demanda atenção especial na testagem empírica de modelos multinível, sendo que as unidades de nível mais elevado devem estar em número suficiente para oferecer a variabilidade necessária à verificação do 
seu efeito. Efeitos topo-descida necessitam de amostragens de mais curto prazo que os fundo-subida. Não existiria uma estratégia única para análise multinível de dados. A seleção dessa estratégia deve ser consistente com os tipos de constructos, modelos, amostragem, dados e questões de pesquisa e com os pressupostos, vantagens e limitações da estratégia. Klein e Koslowski (2000), no entanto, chegam a sugerir várias estratégias estatísticas que poderiam ser úteis: análise de co-variância (ANCOVA); análise de contexto, usando regressão ordinária de quadrados mínimos (OLS); análise de regressão OLS entre níveis e multinível; análises dentro e entre níveis (WABA) e modelos multiníveis de coeficiente aleatório ou randômico (MRCM), tais como modelagem linear hierárquica (HLM) e análise estrutural multinível de covariância (MCSA) ${ }^{(4)}$. Para tanto, o pesquisador conta com diversas opções de programas de análises de dados, tais como o MlwIN, LISREL 8, HLM, SAS, ML3 e VARCL3 - VARCL9.

\section{A Perspectiva Multinível na Compreensão das Transferências nas Organizações}

Na seção anterior, a abordagem multinível para o estudo do comportamento organizacional foi descrita e os exemplos oferecidos foram propositadamente voltados para a questão da aprendizagem e das transferências a ela relacionadas. Na presente seção, esforço adicional será realizado no sentido de enfocar mais especificamente a questão das transferências e de como poderiam ser tratadas, visando à construção de uma teoria multinível.

Koslowski, Brown, Weissbein, Cannon-Bowers e Salas (2000) propõem que na transferência de aprendizagem ocorre um vínculo horizontal entre contextos ocupacionais ou organizacionais: a pessoa adquire CHAs em T\&D e as transfere para seu trabalho. A transferência deste nível individual para o de equipes ou de organização seria vertical ou por emersão e estaria relacionada a processos de outras duas naturezas:

. de composição, baseados no conceito de isomorfismo, havendo similaridade de conteúdos, de relações entre eles e de significados deles nos diferentes níveis;

. de compilação, baseados no conceito de descontinuidade, ocorrendo manifestações dos conteúdos em formas distintas nos diversos níveis.

Quando são examinados os desempenhos do indivíduo e de uma equipe e os 
primeiros contribuem com o mesmo conteúdo de trabalho para os segundos, ainda que varie a quantidade deste conteúdo, este é um caso de composição. Pessoas, trabalhando em equipes, que prestam serviços de atendimento telefônico ao consumidor, são um bom exemplo disto. Mas, se as contribuições dos desempenhos individuais para o desempenho da equipe forem essencialmente distintas, envolvendo CHAs diferentes e conteúdos de desempenho diferenciados, então este é um caso de compilação. Um exemplo poderia ser as equipes das companhias aéreas que trabalham numa aeronave.

A transferência vertical ou emersão seria facilitada na medida em que ocorresse um alinhamento entre as ações de $T \& D$ e a natureza desses processos:

. se composição, as pessoas poderiam aprender em diferentes épocas e eventos;

. se compilação, as pessoas deveriam aprender preferencialmente em conjunto.

Uma análise da natureza dessas condições para transferência vertical deixa evidente que elas são substancialmente organizacionais. Por outro lado, como já foi discutido na primeira seção do presente ensaio, as condições que facilitam a transferência horizontal são essencialmente de aprendizagem, sejam elas prescritas pelas teorias S-R ou S-O-R.

A pesquisa sobre transferência vertical precisaria levar em conta que em processos de composição seria conveniente obter os indicadores de mudanças, nos níveis superiores, em termos de médias ou adições, calculadas com base nos indicadores dos níveis inferiores; mas, em processos de compilação, seria necessário dar atenção, nos níveis superiores, a indicadores de dispersão ou variância dos indicadores dos níveis inferiores. Além disso, neste segundo tipo de processo seria preciso contar, nos níveis superiores, com uma medida independente daquelas dos níveis inferiores e que esta medida fosse global para o nível e não uma combinação de medidas dos níveis inferiores. O desenho da pesquisa para fenômenos de compilação deveria verificar a transferência em vários momentos, enquanto no primeiro caso o desenho poderia ser estático.

Sintetizando, os processos de compilação envolvidos na transferência vertical apresentam várias implicações para o delineamento de pesquisas em avaliação da efetividade de processos de T\&D, em contextos organizacionais. Um foco central é a necessidade de validação de modelos bem especificados, vinculando indicadores de mudança em níveis inferiores àqueles estabelecidos em níveis superiores. Conforme mencionado, o tempo constitui fator crítico no desenvolvimento de processos de compilação ou emersão, que requerem, portanto, para sua investigação, delineamentos de pesquisa longitudinais e a adaptação de sistemas analíticos multiníveis complexos e sofisticados. Uma 
questão metodológica importante diz respeito à seleção da estratégia de amostragem para estudos de avaliação, envolvendo processos compilacionais. Deverão ser incorporadas séries de tempo apropriadas, para que a intervenção tenha efeitos em níveis superiores. A amostragem, portanto, precisa ser sensível às fases críticas em processos de compilação, quando o fenômeno pode mudar para níveis superiores, ou seja, é imprescindível o entendimento dos espaços de tempo necessários ao desenvolvimento e manifestação de diferentes tipos de resultados de compilação.

\section{As Pesquisas Nacionals sobre Transferência nas Organizações}

Esta seção descreve e analisa pesquisas sobre transferência de aprendizagem nas organizações, mostrando como têm sido realizadas as medidas de transferência e identificados os seus preditores. Em seguida, destaca alguns aspectos metodológicos de algumas delas, escolhidas com base no critério da diversidade das medidas e dos preditores investigados. Finalmente, sugere como estudos futuros poderiam levar em conta a abordagem multinível.

A amostra nacional foi composta de 12 trabalhos, em que a transferência de aprendizagem se constitui em variável critério de interesse, mensurada em termos do impacto de treinamento no trabalho.

O Quadro 1, a seguir apresenta esquematicamente as principais características metodológicas e resultados das pesquisas nacionais.

\section{Quadro 1: Pesquisas Nacionais sobre Transferência de Aprendizagem nas Organizações}

\begin{tabular}{|c|c|c|}
\hline Autor/Ano & Metodologia & Resultados Principais \\
\hline \multirow{4}{*}{$\begin{array}{l}\text { Pantoja, M. J., Porto, J. \& } \\
\text { Borges-Andrade, J. E. (2001) }\end{array}$} & \multirow{3}{*}{$\begin{array}{l}\text { Amostra: } 80 \text { profissionais de } \\
\text { nível superior da área de saúde } \\
\text { Tipo de medida: impacto em } \\
\text { amplitude }\end{array}$} & \multirow{4}{*}{$\begin{array}{l}\text { Preditores: } \\
\text { Suporte psicossocial à transferência. } \\
\text { Tipo motivacional } \\
\text { conservação/coletivismo. }\end{array}$} \\
\hline & & \\
\hline & & \\
\hline & $\begin{array}{l}\text { Análise de dados: regressão } \\
\text { hierárquica }\end{array}$ & \\
\hline \multirow[t]{5}{*}{ Sallorenzo. L. H. (2000) } & \multirow{4}{*}{$\begin{array}{l}\text { Amostra: } 1303 \text { servidores de } \\
\text { nível superior do TCU divididos } \\
\text { em amostras A e B. } \\
\text { Tipo de medida: impacto em } \\
\text { amplitude. }\end{array}$} & Preditores: \\
\hline & & Impacto em A \\
\hline & & $\begin{array}{l}\text { Suportes psicossocial e material à } \\
\text { transferência, reação, e motivação. }\end{array}$ \\
\hline & & Impacto em B \\
\hline & $\begin{array}{l}\text { Análise de dados: regressão } \\
\text { múltipla do tipo stepwise }\end{array}$ & $\begin{array}{l}\text { Suportes psicossocial e material à } \\
\text { transferência e reação. }\end{array}$ \\
\hline
\end{tabular}




\section{(continuação)}

\section{Quadro 1: Pesquisas Nacionais sobre Transferência de Aprendizagem nas Organizações}

\begin{tabular}{lll}
\hline Autor/Ano & Metodologia & Resultados Principais \\
\hline Rodrigues, A. G (2000) & $\begin{array}{l}\text { Amostra: } 602 \text { funcionários do } \\
\text { TST-DF } \\
\text { Tipo de medida: impacto em } \\
\text { amplitude. }\end{array}$ & $\begin{array}{l}\text { Preditores: } \\
\text { Suporte psicossocial à transferência, } \\
\text { comprometimento com a carreira, } \\
\text { natureza da participação, lotação e } \\
\text { escolaridade. }\end{array}$ \\
& $\begin{array}{l}\text { Análise de dados: regressão } \\
\text { múltipla do tipo stepwise }\end{array}$ & \\
\hline Pilati, R. \& Borges-Andrade, J. & $\begin{array}{l}\text { Amostra: 210 funcionários e } \\
\text { servidores de duas organizações. } \\
\text { E (2000) }\end{array}$ & $\begin{array}{l}\text { Preditores: } \\
\text { Impacto em amplitude: } \\
\text { amplitude e em profundidade. }\end{array}$ \\
& $\begin{array}{l}\text { Análise de dados: análise de } \\
\text { regressão múltipla stepwise. }\end{array}$ & $\begin{array}{l}\text { Suporte psicossocial à transferência } \\
\text { Impacto em profundidade: }\end{array}$ \\
& $\begin{array}{l}\text { Tempo de organização, suporte } \\
\text { psicossocial à transferência e } \\
\text { escolaridade }\end{array}$ \\
\hline
\end{tabular}

Pilati, R.; Borges-Andrade, J. E. Amostra: 144 participantes de \& Azevedo, L. P. S. (1999)

Pantoja, M. J. (1999) nível médio e de nível superior

Tipo de medida: impactos em amplitude e em profundidade

Análise de dados: regressão múltipla do tipo stepwise

\section{Preditores:}

Impacto em profundidade:

Suporte psicossocial à transferência
Amostra: 345 participantes de nível superior: 263 treinandos e 82 supervisores.

Tipo de medida: impacto em amplitude.

Análise de dados: regressão múltipla do tipo stepwise

\section{Preditores:}

Auto-avaliação de impacto:

Suporte psicossocial à transferência, características individuais e de treinamento

Hetero-avaliação de impacto Suportes psicossocial e material à transferência, características de treinamento

Martins, M. C.; Pinto-Jr, H. \&

Borges-Andrade, J. E. (1999)

Amostra: 52 participantes.

Tipo de medida: impactos em amplitude e em profundidade

\section{Preditor:}

Suporte psicossocial à transferência.

Análise de dados: regressão múltipla do tipo stepwise

Borges-Andrade, J. E.; Gama,

A. L.G.; \& Oliveira-Simões, J.

T. (1999)

\section{Amostra: 629 participante: 411 treinandos e 218 gerentes imediatos}

Tipo de medida: impacto em amplitude

Análise de dados: regressão múltipla do tipo stepwise
Preditores:

Auto-avaliação de impacto:

Satisfação com o desempenho do instrutor e suporte psicossocial à transferência

Hetero-avaliação de impacto:

Suporte psicossocial à transferência e tipo de treinamento 
(conclusão)

\section{Quadro 1: Pesquisas Nacionais sobre Transferência de Aprendizagem nas Organizações}

\begin{tabular}{ll}
\hline Autor/Ano & Metodologia \\
\hline Borges-Andrade, J. E.; Azevedo, & Amostra: 404 participantes - \\
L. P. S.; Pereira, M. H. G. G.; & Auto-avaliação: treinandos \\
Rocha, K. C. P. \& Puente- & Hetero avaliação: chefes ou \\
Palacios, K. E. (1999) & colegas \\
& Tipo de medida: impactos em \\
& amplitude e profundidade \\
& Análise de dados: regressão \\
& múltipla do tipo stepwise
\end{tabular}

Borges-Andrade, J. E.; Morandini, D. C.; \& Machado, M. S. (1999)
Amostra: 159 participantes

Tipo de medida: impactos em amplitude e profundidade

Análise de dados: regressão múltipla do tipo stepwise

\section{Resultados Principais}

Preditores:

Impacto em profundidade:

Satisfação com o trabalho e autonomia da tarefa, carga de trabalho, tipo de treinamento, natureza gerencial da tarefa, suporte psicossocial à transferência, aprendizagem

Impacto em amplitude:

Auto-avaliação:

Aprendizagem, escolaridade, impacto em profundidade

Hetero-avaliação:

Impacto em profundidade, escolaridade, natureza administrativa da tarefa e suporte psicossocial à transferência

Preditores:

Auto-avaliação:

Suporte material à transferência, grau de importância atribuído ao treinamento pelo líder, ocupação de cargo gerencial.

Hetero-avaliação:

Suporte material à transferência

\begin{tabular}{ll}
\hline Abbad, G. (1999). & Amostra: 4051 servidores. A \\
& maioria do TCU, nível superior \\
& completo, divididos em amostras \\
& A e B. \\
& Tipo de medida: impacto em \\
amplitude
\end{tabular}

\section{Impacto em B:}

Análise de dados: regressão múltipla do tipo stepwise

\section{Preditores: \\ Impacto em A:}

Suporte psicossocial à transferência, melhor desempenho, disseminação do curso, entre outros.

Suporte psicossocial à transferência, melhor desempenho grupal, promoções compatíveis, interesses pessoais, esforço melhor desempenho, entre outros.

Borges-Andrade, J. E. \& Siri, C. Amostra: 26 organizações da (1998).

América Latina e Caribe.

Tipo de Medida: impacto em profundidade

\section{Preditores:}

Motivação e capacitação, tanto na auto quanto na hetero-avaliação.

Análise de dados: quantitativa e qualitativa

Os estudos nacionais sobre transferência de aprendizagem apresentam algumas características teóricas e metodológicas bastante claras. Quanto aos aspectos teóricos, emergem dois importantes conceitos: aprendizagem e transferência. No que se refere à aprendizagem, sua ocorrência manifesta-se no nível individual 
e, conforme discutido na primeira seção do presente ensaio, o termo envolve a noção de que a experiência de interação do indivíduo com seu ambiente (S) lhe possibilitaria apreender algo $(\mathrm{O})$ - uma capacidade (por exemplo, um conceito ou uma forma de resolver um problema); ou uma disposição (por exemplo, um interesse ou um valor), que seria futuramente mostrado, manifestado, evidenciado ou revelado por meio de alguma mudança em seu comportamento (R). Se a mudança no indivíduo ocorre em atividades não equivalentes às anteriores ou é verificada em situações distintas daquelas em que ocorreu a aquisição, pode ser dito que ocorreu uma transferência de aprendizagem.

O foco central das pesquisas nacionais descritas envolve processos de transferência horizontal, tipicamente investigados no nível individual. Isto significa que os resultados obtidos em tais estudos podem somente ser analisados para compreender as transformações que acontecem no desempenho do indivíduo no trabalho, mas não o que ocorre nos níveis de equipe e de organização. Desta forma, enquanto que na transferência de aprendizagem a pessoa adquire CHAs em T\&D e as transfere para seu trabalho, na transferência deste nível individual para o de equipes ou de organização ocorre um vínculo vertical ou por emersão e está relacionada a processos de outras duas naturezas: de composição e de compilação e pode ser facilitada na medida em que ocorra alinhamento entre ações de T\&D e a natureza desses processos. Nesta perspectiva, transferência horizontal e transferência vertical envolvem questões de naturezas distintas: transferência horizontal, que é por natureza de aprendizagem; e vertical, cuja natureza é organizacional.

Os modelos de investigação utilizados são bidimensionais e buscam examinar as relações entre vários constructos no mesmo nível, não se propondo, entretanto, a investigar os relacionamentos recíprocos entre constructos em diferentes níveis de análise. Geralmente, são pesquisas correlacionais de campo que investigam o relacionamento entre transferência de aprendizagem e variáveis relativas às características individuais do treinando, características do projeto de treinamento e de suporte e clima por meio de medidas de natureza perceptual e auto-avaliações tomadas no período de tempo mínimo de duas semanas e máximo de quatro anos após o término do treinamento.

No que tange à variável tempo, tão importante em delineamentos de pesquisas na área de transferência vertical, especialmente em processos de compilação, observa-se que os estudos nacionais, em função de focalizarem a transferência horizontal, tendem a concentrar a coleta de dados em períodos relativamente curtos de tempo (tempo médio de 4 meses) e em geral apenas no final do curso. Tal situação tenderia a mudar radicalmente em contexto de pesquisa com foco na transferência vertical, que exige delineamentos de pesquisa longitudinais e muita atenção na especificação das séries de tempo para coleta de dados. 
As pesquisas nacionais utilizaram dois tipos de medidas de transferência de aprendizagem: medidas em amplitude e em profundidade. A transferência de aprendizagem em amplitude diz respeito aos efeitos gerais do evento instrucional sobre o desempenho de tarefas relacionadas, diretamente ou não, aos conteúdos aprendidos no curso, enquanto a transferência de aprendizagem em profundidade mensura o efeito das ações de T\&D em tarefas estritamente relacionadas aos conteúdos específicos, ensinados nos curso.

Tais medidas utilizadas nos modelos de investigação de pesquisas nacionais em transferência de aprendizagem, conforme Abbad, Pantoja e Pilati (2001), precisam incluir em seus conceitos noções de verticalidade e horizontalidade, à luz da abordagem multinível, bem como revisões conceituais de suas variáveis preditoras nos níveis superiores, para que possam ser incorporadas em modelos tridimensionais mais complexos e sofisticados.

Quanto aos principais preditores da transferência de aprendizagem nas organizações, destacam-se variáveis relativas às características individuais dos ex-treinandos, características do treinamento e de suporte e clima organizacional à transferência, sendo que a variável que apresentou maior poder de explicação da transferência de aprendizagem nas organizações estudadas foi o suporte psicossocial. Tais dados fornecem evidências de que as condições que facilitam a ocorrência da transferência horizontal são, de fato, essencialmente "de aprendizagem".

A seguir, serão analisadas mais especificamente duas pesquisas nacionais na área de transferência de aprendizagem, selecionadas em função da diversidade de medidas e preditores utilizados.

O modelo de avaliação de transferência de aprendizagem, desenvolvido e testado por Rodrigues (2000), investigou a relação entre comprometimento organizacional, caraterísticas individuais e natureza da participação no treinamento (espontânea ou obrigatória) e transferência de aprendizagem. Os resultados indicaram que ex-treinandos com elevados níveis de comprometimento organizacional com a carreira relataram mais intensamente os impactos de treinamentos em seu desempenho individual e que estes relatos estão fortemente associados a percepções de suporte psicossocial à transferência do aprendido no trabalho. Tal estudo poderia ser redesenhado dentro de um enfoque multinível, visando examinar se variáveis do nível do grupo ou da organização agregariam maior poder preditivo, bem como possibilitariam melhor ajustamento do modelo aos dados. Para tanto, torna-se necessário considerar o fenômeno da emergência no que se refere aos constructos suporte organizacional à transferência e comprometimento organizacional. Neste sentido, seria necessária a redefinição de tais contructos 
nos níveis de grupo e organização, bem como o desenvolvimento e construção de medidas, a partir dos novos conceitos adotados. Além disso, poderia ser ainda, verificado o efeito moderador exercido pelo tipo de participação no treinamento, voluntária ou obrigatória, na relação entre comprometimento e transferência de aprendizagem.

Pantoja, Porto e Borges-Andrade (2001) focalizaram a influência exercida pelos valores individuais do treinado e suas percepções de suporte à transferência na explicação da transferência de aprendizagem no trabalho. Emergiram como preditores suporte psicossocial e valores individuais do tipo conservação/coletivismo. Em perspectiva multinível, haveria necessidade de aumento da amostra, tendo em vista que foi constituída de 80 casos; e ainda, uma especificação das séries de tempo para coleta de dados. Poderiam ser acrescentados ao modelo hipotetizado constructos no nível da organização, como, por exemplo, valores organizacionais, bem como investigadas suas relações com as percepções de suporte psicossocial no nível do grupo. Seria interessante, ainda, investigar o efeito moderador exercido pela variável tipo de treinamento no relacionamento entre valores individuais e transferência de aprendizagem.

Sintetizando, de maneira geral as pesquisas nacionais constituem estudos clássicos na área de transferência de aprendizagem nas organizações e focalizam predominantemente os processos de transferência horizontal. Ainda que investiguem a influência de fatores contextuais na explicação da transferência de aprendizagem, fazem-no sem considerar suas possíveis representações em níveis superiores como grupos e organização, bem como suas relações com os demais níveis do sistema organizacional.

Cabe ressaltar que a maioria das lacunas identificadas nas pesquisas analisadas decorrem do fato de que tais estudos não foram originalmente desenhados dentro de uma perspectiva multinível. Para tanto, torna-se necessário que sejam contempladas importantes questões conceituais e metodológicas. Com relação aos aspectos conceituais, é importante a redefinição de diversos constructos como comprometimento, suporte psicossocial à transferência, motivação, auto-eficácia, entre outros, em diferentes níveis do sistema organizacional. No que tange aos aspectos metodológicos, é indispensável e urgente a utilização de procedimentos de amostragem que permitam a variabilidade necessária entre as unidades, em todos os níveis relevantes do modelo. Além disso, que sejam incorporadas análises dos efeitos de variáveis contextuais em diferentes sentidos: do organizacional para o individual e do individual para o organizacional. 


\section{Conclusões}

Este ensaio teve como objetivos analisar os conceitos de aprendizagem e de transferência de aprendizagem nas organizações e sintetizar os princípios da abordagem multinível para a construção de teoria e a realização de pesquisa nas organizações, bem como demonstrar como eles podem ser aplicados para a compreensão da transferência de aprendizagem decorrente dos processos de T\&D. Além disso, a partir de um levantamento da produção nacional de pesquisas sobre transferência de aprendizagem nas organizações, mostrar como têm sido realizadas as medidas de transferência e identificados os seus preditores.

A análise das pesquisas nacionais sobre transferência de aprendizagem forneceu evidências de que os modelos de investigação utilizados ainda são bidimensionais e envolvem somente processos de transferência horizontal, que focalizam tipicamente o nível individual.

No que se refere à perspectiva multinível, cabe ressaltar a importância das questões conceituais para o delineamento de modelos mais articulados de investigação que assumam implicitamente vínculos existentes entre os níveis individual, do grupo e da organização. Este enfoque, de acordo com Koslowski,, Brown, Weissbein, Cannon-Bowers e Salas (2000) é apenas um ponto de partida para que maiores desenvolvimentos teóricos e metodológicos ocorram na área da efetividade de processos de T\&D e da organização como um todo. De fato, muitos são os desafios das pesquisas futuras sobre transferência de aprendizagem, entre os quais a incorporação de processos horizontais e verticais, bem como a análise dos níveis de grupo e organização. Além disso, há a análise das ligações entre os diferentes níveis, em seus relacionamentos recíprocos. Os novos modelos de investigação devem incluir também a análise dos efeitos de variáveis contextuais em diferentes sentidos: do organizacional para o individual e do individual para o organizacional e nos diferentes níveis, envolvendo indivíduo, grupo e organização.

\section{Notas}

\footnotetext{
${ }^{1}$ Para uma análise conceitual mais aprofundada do termo "aprendizagem" consultar, por exemplo, Howard (1999), que chega ao extremo de argumentar pelo abandono do termo, por causa da confusão conceitual que o rodeia. Contudo, ao sugerir que este seja substituído pela noção de criação e mudança de representações mentais, o autor introduz outro termo que é igualmente cercado de confusão conceitual.
} 
${ }^{2}$ Este mútuo desconhecimento foi recentemente verificado pelo segundo autor do presente texto, durante visita pessoal a Birdi, quando conversou sobre as coincidências entre ambas propostas.

${ }^{3}$ Ver Klein e Koslowski (2000), que definem essas categorias e seus componentes e apresentam exemplos.

${ }^{4}$ O uso dessas estratégias, para análise de dados multiníveis, é descrito e discutido em detalhes por Bliese (2000), Dansereau e Yammarino (2000), Hofmann, Griffin e Gavin (2000), James e Williams (2000) e Klein, Bliese, Kozlowski, Dansereau, Gavin, Griffin, Hofmann, James, Yammarino e Bligh (2000).

\section{Artigo recebido em 12.02.2003. Aprovado em 07.06.2004.}

\section{ReferÊnCIAS Bibliográficas}

\section{ABBAD-OC, G.}

Um modelo integrado de avaliação do impacto do treinamento no trabalhoIMPACT. 1999. 262f. Tese (Doutorado em Psicologia) - Universidade de Brasília. Brasília: 1999.

ABBAD, G;

BORGES-ANDRADE, J.E.

Aprendizagem humana em organizações de trabalho. In: J. C. ZANELLI; J.E. BORGES-ANDRADE; A. V. B. BASTOS (Ed.): Psicologia, organizações e trabalho no Brasil. Porto Alegre: ARTMED, 2004. p. 237275.

\section{ABBAD, G; \\ PANTOJA, M. J.; \\ PILATI, R.}

Preditores de efeitos de treinamento: o estado da arte e o futuro necessário. In: ENCONTROANUALDAANPAD, 25., 2001. Campinas. Anais... Campinas: ANPAD, 2001. v. CD-Rom.

\section{ABBAD, G. et al.}

Preditores de impacto do treinamento no trabalho - o caso do TCU (Resumo). In: Sociedade Brasileira de
Psicologia (Org.), Resumos de Comunicação Científica, XXIX Reunião Anual de Psicologia. Ribeirão Preto: SBP. 1999. 53 p.

BALDWIN, T. T.;

FORD, J. K.

Transfer of training: a review and directions for future research. Personnel Psychology, [ S.L.], v. 41, n. 1, p. 63-105. April 1988.

BIRDI, K.

The bigger picture: identifying the factors influencing training effectiveness. 2000. Trabalho apresentado no seminário Working to Learn, Sheffield, UK: ESRC/University of Sheffield, 2000. Não publicado.

BLIESE, P.D.

Within-Group agreement, nonindependence, and reliabilitity: implications for data aggregation and analysis. In: K. J. KLEIN; S. W. J. KOSLOWSKI(Ed.): Multilevel theory, research, and methods in organizations: foundations, extensions, and new directions. San Francisco: Jossey-Bass. 2000. p. 349-381. 
BORGES-ANDRADE, J. E.

Desenvolvimento de medidas em avaliação de treinamento. Estudos de Psicologia, v. 7, número especial, p. 31-43. 2002.

BORGES-ANDRADE, J. E. et al.

Impacto de treinamento no trabalho: o caso do Banco do Brasil. In: REUNIÃO ANUAL DE PSICOLOGIA, 29., 1999 Ribeirão Preto. Resumos... Ribeirão Preto: 1999, Ribeirão Preto: Sociedade Brasileira de Psicologia. p. 53C.

\section{BORGES-ANDRADE, J.E.;}

GAMA, A.L. G.;

OLIVEIRA-SIMÕES, J. T.

Impacto do treinamento no trabalho: um estudo de caso na Eletronorte. In: REUNIÃO ANUAL DE PSICOLOGIA, 29., 1999, Ribeirão Preto.

Resumos... Ribeirão Preto: Sociedade Brasileira de Psicologia, 1999. p. 53b53c.

BORGES-ANDRADE, J.E.;

MORANDINI, D. C.;

MACHADO, M. S.

Impacto de treinamento gerencial e efetividade de equipes em ambientes de inovação tecnológica. In: REUNIÃO ANUAL DE PSICOLOGIA, 29., 1999, Ribeirão Preto. Resumos... Ribeirão Preto: Sociedade Brasileira de Psicologia, 1999. p. 53b-53c.

\section{BORGES-ANDRADE, J.E.;}

SIRI, C.

Impacts of the PM-\&-E project's training activitites (Abstract). In: D. HORTON, L. DUPLEICH; A. ANDERSON (Org.), Assessing organizational impact. Reports of review and synthesis workshop. The Hague, Netherlands: International Service for National Agricultural Research. 1998. p. 5.

DANSEREAU, F.;

YAMMARINO, F. J.

Within and Between Analysis: the variant paradigm as an underlying approach to theory building and testing. In: K. J. KLEIN; S. W. J. KOSLOWSKI (Ed.): Multilevel theory, research, and methods in organizations: foundations, extensions, and new directions. San Francisco: Jossey-Bass. 2000. p. 425466.

\section{GAGE, N. L.;}

BERLINER, D. C.

Educational psychology. Boston: Houghton Mifflin. 1984.

\section{GAGNÉ, R. M.;}

DRISCOLL, M. P.

Essentials of learning for instruction. Englewood Cliffs, N. J.: Prentice Hall. 1988.

HOFMANN, D. A.; GRIFFIN, M.A.;

GAVIN, M. B.

In: KLEIN, K. J.; KOSLOWSKI, S. W. J. (Ed.): Multilevel theory, research, and methods in organizations: foundations, extensions, and new directions. San Francisco: JosseyBass, 2000. p. 467-511

HOWARD, R. W.

Reconceptualizing learning. Review of General Psychology, [S.L.], v. 3 n. 4, p. 251-263. Oct.1999. 
JAMES, L. R.;

WILLIAMS, L. J.

The cross-level operator in regression, ANCOVA, and contextual analysis. In: KLEIN, K. J.; KOSLOWSKI, S. W. J. (Ed.): Multilevel theory, research, and methods in organizations: foundations, extensions, and new directions. San Francisco: JosseyBass. 2000. p. 382-424.

KLEIN, K. J. et al.

In: KLEIN, K. J.; KOSLOWSKI, S. W. J. (Ed.): Multilevel theory, research, and methods in organizations: foundations, extensions, and new directions. San Francisco: JosseyBass. 2000. p. 512-553.

KLEIN, K. J.;

KOSLOWSKI, S. W. J.

Multilevel theory, research, and methods in organizations: foundations, extensions, and new directions. San Francisco: JosseyBass. 2000.

KOSLOWSKI, S. W. J. et al.

A multilevel approach to training effectiveness: enhancing horizontal and vertical transfer. In: KLEIN, K. J.; KOSLOWSKI, S. W. J. (Ed.): Multilevel theory, research, and methods in organizations: foundations, extensions, and new directions. San Francisco: JosseyBass. 2000. p. 157-210.
KOSLOWSKI, S. W. J.;

KLEIN, K. J.

A multilevel approach to theory and research in organizations: contextual, temporal, and emergent processes. In: KLEIN, K. J.; KOSLOWSKI, S. W. J. (Ed.): Multilevel theory, research, and methods in organizations: foundations, extensions, and new directions. San Francisco: JosseyBass. 2000. p. 3-90.

\section{PANTOJA, M. J.}

Avaliação de impacto de treinamento na área de reabilitação: preditores individuais e situacionais. 1999. 106 f. Dissertação (Mestrado em Psicologia) - Universidade de Brasília, Brasília.

PANTOJA, M. J.;

PORTO, J.;

BORGES-ANDRADE, J.E.

Valores individuais, suporte à transferência e impacto do treinamento no trabalho. In: CONGRESSO INTERAMERICANO DE PSICOLOGIA, 28., 2001, Santiago -Chile. Anais... Santiago - Chile: SIP, 2001. v. CD-Rom.

PILATI, R.;

BORGES-ANDRADE, J.E.;

AZEVEDO, L.P.S.

Impacto do treinamento em amplitude e profundidade: relações com suporte à transferência, gestão do desempenho e liberdade decisória. In: REUNIÃO ANUAL DE PSICOLOGIA, 29., 1999. Ribeirão Preto. Resumos... Ribeirão Preto: Sociedade Brasileira de Psicologia, 1999. p. 53. 
RODRIGUES, A. G. A

Natureza da participação e suas implicações no impacto do treinamento no trabalho. 2000. $112 \mathrm{f}$.

Dissertação (Mestrado em Psicologia)

- Universidade de Brasília, Brasília.

SALAS, E.;

CANNON-BOWERS, J.

The science of training: a decade of progress. Annual Review of Psychology, v. 52, 2001. p. 471-499.
SALLORENZO.L.H.

Avaliação de impacto de treinamento no trabalho: analisando e comparando modelos de predição. 2000. 66 f. Dissertação (Mestrado em Psicologia) - Instituto de Psicologia, Universidade de Brasília, Brasília. 